\section{EPITHELIAL TO MESENCHYMAL TRANSITION}

\section{Layers of regulation}

Epithelial to mesenchymal transition (EMT), which can enhance the migration and invasion of cancer cells, is known to be regulated transcriptionally, but less is known about other mechanisms that regulate this process. Two recent papers have shown that EMT can be driven by both alternative splicing and chromatin modification.

Using RNA-Seq to analyse global changes in mRNA splicing in a human mammary epithelial cell line (HMLE cells) expressing the EMTassociated transcription factor twist, Christopher Burge, Frank Gertler and colleagues found a substantial change in splicing. Many of the genes that were alternatively spliced during EMT were involved in processes such as cell migration, actin cytoskeletal regulation and cell-cell junction formation, all of which contribute to phenotypic changes that occur during EMT. Most splicing factors bind to short sequences of RNA, and the authors found an enrichment of binding motifs for the RNA binding

EMT can be driven by both alternative splicing and chromatin modification. tein (ESRP) families of splicing factors near alternative exons, the usage of which changed following EMT. ESRP1 expression decreased during data, either expression of ESRP1 or knockdown of RBFOX2 in HMLE cells expressing twist led to more epithelial-specific splicing events and partially reverted the morphological, junctional and migratory phenotypes of these cells from mesenchymal to epithelial. Furthermore, the examination of a panel of human breast cancer cell lines indicated that aggressive and metastatic cells (of a basal-like origin) had more EMTassociated alternative splicing events than cells that were poorly metastatic (of a luminal origin), and the cell lines could be correctly classified using this alternative splicing signature. Alternative mRNA isoforms identified in the breast cancer cell lines were also expressed in primary invasive ductal carcinomas, which tended to have either epithelial or mesenchymal splicing patterns, but not both. These data suggest the importance of alternative splicing in directing EMT and also the possibility of using alternative splicing signatures as prognostic or diagnostic markers for breast cancer.

Kou-Juey Wu and colleagues used a bioinformatic approach to search for chromatin modifiers that are involved in hypoxia-induced EMT, and they identified histone deacetylase 3 (HDAC3) as a direct transcriptional EMT, whereas RBFOX2 expression increased; consistent with these

$$
\text { (1) }
$$

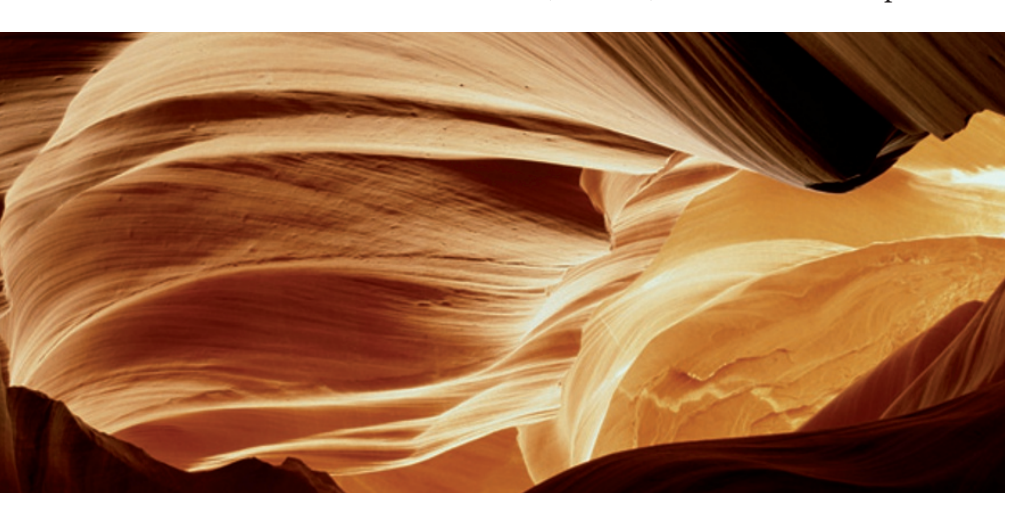

target of hypoxia-inducible factor 1 (HIF1). Small interfering RNA (siRNA) against HDAC3 in $\mathrm{FaDu}$ hypopharyngeal carcinoma cells overexpressing a constitutively active mutant of HIF1a and H1299 lung cancer cells (which constitutively express wild-type HIF1) indicated that HDAC3 is crucial for HIF1 $\alpha$-mediated EMT and the induction of metastatic phenotypes. HDAC3, together with WDR5 (a component of the histone methyltransferase (HMT) complex), decreased global acetylation of histone H3 lysine 4 (H3K4Ac) and increased methylation of H3K4 (H3K4me2 and H3K4me3) in FaDu or MCF7 breast cancer cells under hypoxic conditions. Sequential quantitative chromatin immunoprecipitation assays indicated that HDAC3 recruited WDR5 to the promoters of mesenchymal genes and specifically increased $\mathrm{H} 3 \mathrm{~K} 4$-specific HMT activity. HDAC3 was also recruited to the promoter of the epithelial-specific gene E-cadherin $(\mathrm{CDH} 1)$ and could enhance repression of $C D H 1$ transcription by the transcription factors snail and twist 1. Furthermore, in patients with head and neck squamous cell carcinomas, those with tumours that had increased expression of HIF1 $\alpha$, HDAC3 and WDR5 had a significantly worse prognosis than those with tumours that did not express these proteins. mRNA levels of these three genes also showed prognostic value in patients with oral cancer.

Understanding these additional levels of regulation of EMT might aid in the development of therapeutics to prevent metastasis, as well as diagnostic or prognostic markers for various tumour types.

Sarah Seton-Rogers

ORIGINAL RESEARCH PAPERS Shapiro, I. M. et al. An EMT-driven alternative splicing program occurs in human breast cancer and modulates cellular phenotype. PLoS Genet. 7, e1002218 (2011) |Wu, M. Z. et al. Interplay between HDAC3 and WDR 5 is essential for hypoxia-induced epithelial-mesenchymal transition. Mol. Cell 43 811-822 (2011) 